Theoretical values of the energy of cohesion and the compressibility were computed from the appropriate potential energy curves which agreed satisfactorily with the experimental values.

\section{ACKNOWLEDGMENTS}

The authors wish to express their thanks to Violet Minchak for performing the calculations and for many valuable suggestions.

THE JOURNAL OF CHEMICAL PHYSICS

VOLUME 25, NUMBER 4

OCTOBER, 1956

\title{
Paramagnetic Resonance Absorption in Some Organic Biradicals
}

\author{
H. S. JARRETT, Chemical Department, Experimental Station, E. I. du Pont de Nemonts and Company, \\ Wimington, Delaware \\ AND \\ G. J. SloaN* $\dagger$ AND W. R. VaughaN, Department of Chemistry, University of Michigan, \\ Ann Arbor, Michigan
}

(Received December 13, 1955)

\begin{abstract}
Four compounds of the form $4,4^{\prime}$-polymethylenebistriphenylmethyl, one compound of the form $(1,4-$ phenylene) bisdiarylmethyl, three compounds of the form $\left(4,4^{\prime}\right.$-biphenylene) bisdiarylmethyl, and one compound, $4,4^{\prime}$-oxybistriphenylmethyl have been shown to possess unpaired electrons by paramagnetic resonance absorption. The resonance spectra of $0.01 \mathrm{M}$ solutions of these compounds in benzene exhibit a hyperfine structure arising from a spherically symmetrical contribution of the magnetic dipole interaction between the unpaired electron and the nuclear magnetic moments of the hydrogen atoms. The $g$-factors for the compounds investigated in the first three classes were found to be $2.0025 \pm 0.0004$ and $2.0031 \pm 0.0004$ for the last compound. Such a close approach of the $g$-factor to the free electron value plus the sharpness of the hyperfine structure lines indicates that the anisotropic contributions of the spin-orbit interaction, which would normally lift the degeneracy of the triplet state, are averaged out by the tumbling of the molecules.
\end{abstract}

\section{INTRODUCTION}

$\mathrm{C}^{\mathrm{B}}$ ERTAIN organic compounds exhibit a chemical behavior characteristic of free radicals but contain even numbers of electrons. ${ }^{1}$ Among these are the $4,4^{\prime}$-polymethylenebistriphenylmethyl radicals and the $p$-substituted polyphenyls.
A.

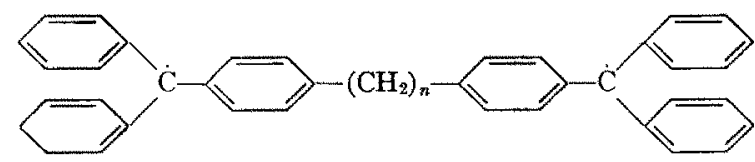

B.<smiles></smiles>

Such compounds would be expected to show a paramagnetic susceptibility caused by the presence of two unpaired electrons. Magnetic susceptibility studies ${ }^{2}$ indicate, however, that many of these compounds are diamagnetic. Two possibilities have been advanced to explain this apparent anomaly : (1) in compounds of the type $B$, the energy separation of the singlet (in which all electrons are paired) from the triplet state (in which two electrons are unpaired) may be so large

* Present address: Chemical Department, Experimental Station, E. I. du Pont de Nemours and Company, Wilmington, Delaware.

Abstracted in part from the Ph.D. dissertation of Gilbert J. Sloan, University of Michigan, 1953.

${ }^{1}$ V. Richter, The Chemistry of the Carbon Compounds (Elsevier Publishing Company, New York, 1947), Vol. IV.

${ }^{2}$ E. Müller, Fortschr. chem. Forsch. 1, 325 (1949)

${ }^{3}$ C. A. Hutchison et al., J. Chem. Phys. 20, 1485 (1952). that the contribution of the triplet to the susceptibility at room temperature may be obscured by the diamagnetism of the molecule; (2) there is no reliable means for determining the actual diamagnetism of the biradical. Recently, Wheland ${ }^{4}$ and Selwood and Dobres ${ }^{5}$ have challenged the conclusions based on the susceptibility studies of these compounds. They have proposed that an anomalously large diamagnetism which may be associated with free radicals could mask the paramagnetic component of the susceptibility.

It therefore seemed desirable to make use of paramagnetic resonance, which is unaffected by the diamagnetism of the molecule, for the determination of the presence of unpaired electrons.

\section{EXPERIMENTAI}

\section{A. Compounds Investigated}

Solutions of the following compounds in benzene were investigated by the resonance method:

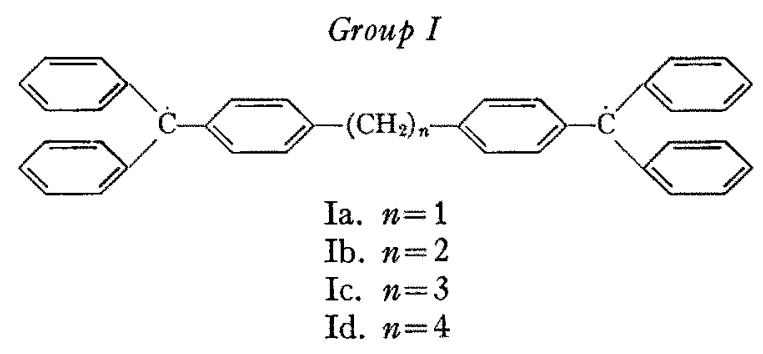

${ }^{4}$ G. W. Wheland, Adwanced Organic Chemisiry (John Wiley \& Sons, Inc, New York, New York, 1949).

${ }^{5}$ P. W. Selwood and R. M. Dobres, J. Am. Chem. Soc. 72, 3860 (1950). 


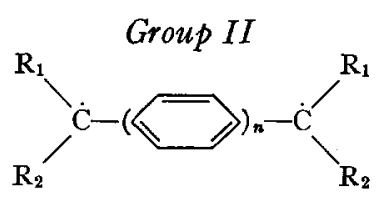

where $n=1$.

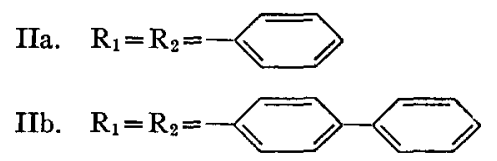

and where $n=2$.
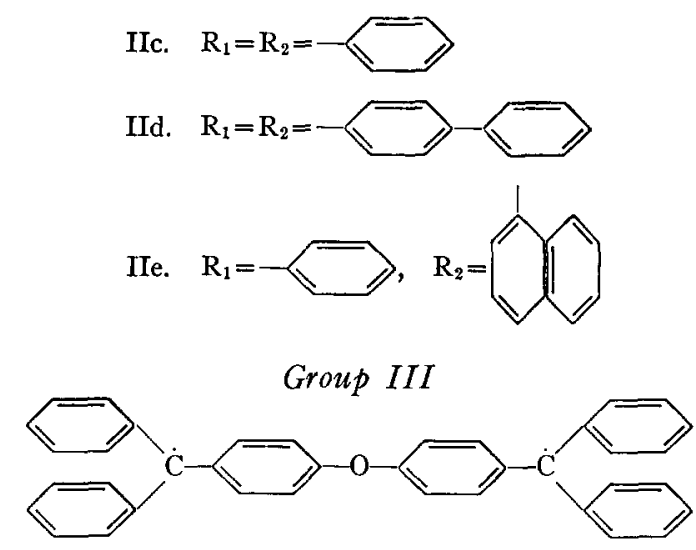

These compounds were prepared in vacuum by the dehalogenation of their dichlorides with finely divided silver. The dichlorides and their free radicals were prepared as described elsewhere. ${ }^{6}$ A quantity of the halide and a tenfold excess of the silver were placed in the upper portion of the reaction vessel, Fig. 1. The volume of dry, gas-free benzene required to make a $0.01 \mathrm{M}$ solution was distilled into the vessel, which had been evacuated to $10^{-6} \mathrm{~mm} \mathrm{Hg}$ by a mercury diffusion pump. The reaction vessel was then sealed from the vacuum system at the constriction. The suspension was shaken in the dark for a few minutes and the benzene solution of the biradical was filtered through a glass frit into the thin-walled capillary. The sample tubes were stored in the dark between measurements. Recovery of silver chloride from a number of the radical

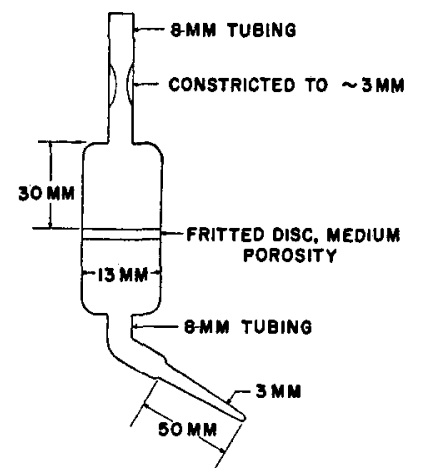

FIG. 1. Reaction vessel in which the dichlorides of the free radicals were dehalogenated. The $3 \mathrm{~mm}$ tip of the vessel was inserted into the resonant cavity.

${ }^{6}$ G. J. Sloan and W. R. Vaughan, J. Org. Chem. (to be published). solutions showed that $84-99 \%$ dehalogenation had occurred.

\section{B. Microwave Apparatus}

Paramagnetic resonance absorption spectra were obtained at $1.2-\mathrm{cm}$ wavelength with standard equipment. The thin-walled tip of the reaction vessel was placed in the center of a cylindrical reflection cavity operated in the $\mathrm{TE}_{011}$ mode. The magnetic field was modulated at 4000 cps with an amplitude (0.1 oersted) which was less than the width of the individual hyperfine structure lines. When resonance absorption occurred, a microwave signal reflected from the resonant cavity contained a $4000 \mathrm{cps}$ modulation component whose amplitude was proportional to the derivative of the absorption. The $4000 \mathrm{cps}$ component was amplified by a phase-sensitive amplifier and recorded as a function of magnetic field strength on a strip chart recorder. A feedback system was employed to stabilize the klystron on the resonant frequency of the sample cavity. The line widths of the absorption spectra were taken as the separation between the positions of maximum slope of a curve constructed by joining the average deviations of the amplitudes of the derivatives of each hyperfine structure line. The $g$-values were obtained by observing the resonance absorption of a small sample of powdered 1,1-diphenyl-2-picrylhydrazyl simultaneously with the resonance absorption of the biradical. The $g$-value is then found from the difference between the fields for maximum resonance absorption of the sample and the hydrazyl line using the value $g=2.0038 \pm 0.0003$ for hydrazyl. ${ }^{7}$ The minimum number of free spins detectable is $5 \times 10^{15} / \mathrm{cc}$ for the hydrazyl in benzene solution. This leads to a minimum detectable triplet state population of $0.01 \%$ at $0.01 \mathrm{M}$ concentration of the compounds. However, since solutions of the biradicals exhibit a narrower line width than the hydrazyl in solution, a smaller number of free spins should be detectable.

\section{RESULTS}

\section{A. Magnetic Resonance Spectra of Group I Compounds}

Figure 2 shows the derivatives of the resonance absorption spectra of the 4,4'-polymethylenebistriphenylmethyl radicals. The spectra of these radicals exhibited a structure which is probably hyperfine, arising from a magnetic dipole interaction between the odd electrons and the hydrogen nuclei. ${ }^{8}$ Therefore, the absorption line widths quoted in Fig. 2 arise almost entirely from these magnetic interactions. The $g$-factors

${ }^{7}$ Holden, Kittel, Merritt, and Yager, Phys. Rev. 77, 147 (1950); C. H. Townes and J. Turkevich, Phys. Rev. 77, 148 (1950).

${ }^{8}$ H. S. Jarrett and G. J. Sloan, J. Chem. Phys. 22, 1783 (1954); B. Venkataraman and G. K. Fraenkel, J. Am. Chem. Soc. 77, 2707 (1955). 
for these compounds were found to be $2.0025 \pm 0.0004$, which is, within experimental error, the $g$-factor for a free electron. Since the phenylene rings are separated by one or more methylene groups, little interaction between the two unpaired electrons is expected. Thus, the biradicals may be considered to possess two independent unpaired electrons and each half of the biradical will give rise to the same absorption spectrum. Therefore, these spectra would be expected to be similar to the spectrum of a triphenylmethyl monoradical that possessed a single para-substituent. It is to be noted, however, that for increased methylene chain length the line width of the individual hyperfine structure lines increases, resulting in reduced resolution of the fine structure in the absorption spectrum. Since the energy levels of the unpaired electron probably do not change greatly with increased chain length, the broader hyperfine structure lines may be caused by incomplete averaging out of the anisotropic component of the hyperfine interaction. That is, the tumbling rate may be comparable to the hyperfine splitting frequency ${ }^{9}$ either because of the larger moment of inertia of the longer molecules or because the molecules become "locked in" to the short range order in the benzene solvent.

All the compounds in group I decompose by disproportionation, ${ }^{10}$ dimerization, or polymerization, since the resonance finally disappears several days after dehalogenation. It is noteworthy that a slight rearrangement of the positions of the hyperfine structure peaks occurs during the degradation. This change is most noticeable in the methylene derivatives (Ia) whose hyperfine structure lines are initially most clearly resolved. The separation of the hyperfine structure peaks decreases slightly during degradation for the compounds with an odd number of methylene groups, netic resonance absorption spectra of the $4,4^{\prime}$-polymethylmethyl radicals.
FIG. 2. Paramagenebistriphenyl-

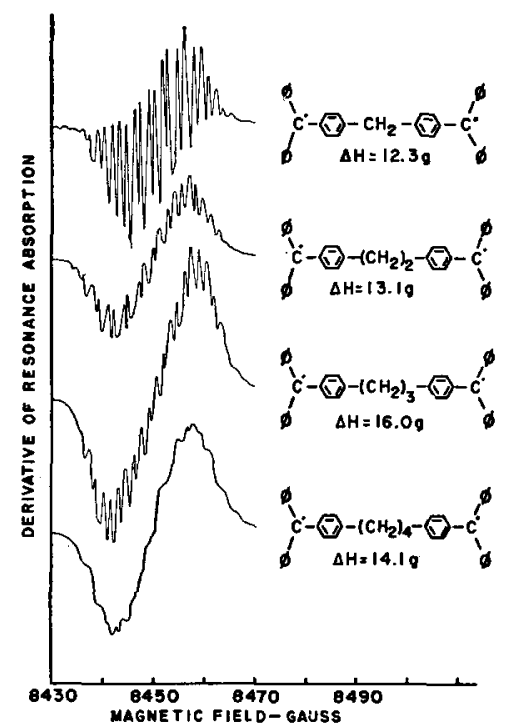

${ }^{9}$ S. I. Weissman, J. Chem. Phys. 22, 1378 (1954).

$10 \mathrm{~J}$. Schmidlin and A. Garcio-Banus, Ber. deut. chem. Ges. 45 1344 (1912); E. Müller, Angew. Chem. 51, 662 (1938).
Fig. 3. Paramagnetic resonance absorption spectra of the $\left(4,4^{\prime}\right.$-biphenylene) bisdiarylmethyl radicals.

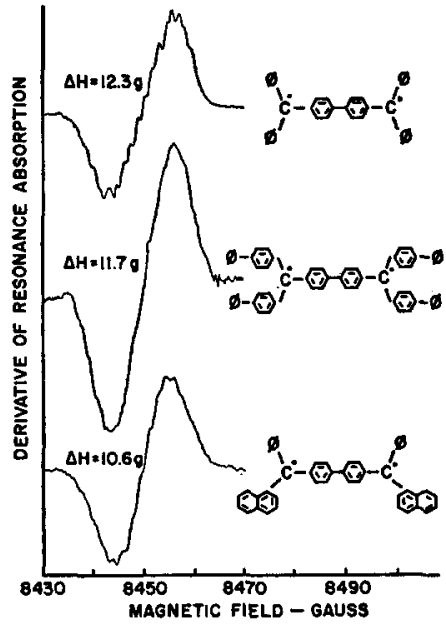

while there is an increase in separation for those compounds with an even number of methylene groups. The reason for this variation is not clear.

\section{B. Magnetic Resonance Spectra of Group II Compounds}

The derivatives of the resonance absorption spectra for the $p$-substituted polyphenyls are shown in Fig. 3 . Barely resolved hyperfine structure also appeared for these compounds. The hyperfine structure line width is comparable to that in the spectrum of the diphenylbutane derivative (Id) in group I. Groups other than phenyl, e.g., xenyl (IId) or $\alpha$-naphthyl (IIe), on the methyl carbon atoms cause the resolution of the hyperfine structure to decrease further. It is difficult to state whether additional hyperfine structure from the hydrogen nuclei of the large aromatic groups reduces the resolution in the spectrum or whether the broadening is caused by incomplete averaging of the anisotropic interactions as the molecule tumbles.

Hutchison ${ }^{3}$ found that in compound IIc the unpaired electrons are associated with a triplet state which lies $2.5 \mathrm{kcal}$ above the diamagnetic ground state. This value agrees very well with the value of $2.9 \mathrm{kcal}$ calculated by the simple MO method.

The $g$-factors for the compounds of group II, where $n=2$, were also found to be $2.0025 \pm 0.0004$. This result is surprising in view of the fact that the unpaired electrons are probably associated with the triplet state. It would be expected that spin-orbit coupling would lift the threefold degeneracy of the triplet energy level ${ }^{11}$ and that magnetic dipole transitions, if observed, would occur at an effective value of $g$ other than 2 .

At first it was thought that the spins of the two electrons might be decoupled by essentially free rotation about the $\sigma$-bond connecting the two phenylene rings. Results of $\mathrm{MO}$ calculations shown below indicate,

11 C. A. Coulson, Valence (Oxford University Press, London, England, 1952), p. 137. 
TABLE I.

\begin{tabular}{ccccc}
\hline \hline & $\begin{array}{c}\text { Ground } \\
\text { state }\end{array}$ & Triplet & & $S_{ \pm}$ \\
\hline$D_{2 h}$ & ${ }^{1} A_{g}$ & ${ }^{3} B_{1 u}$ & ${ }^{1} B_{3 u},{ }^{1} B_{2 u}$ & $S_{z} A_{u}$ \\
$D_{2}$ & ${ }^{1} A$ & ${ }^{3} B_{1}$ & ${ }^{1} B_{3},{ }^{1} B_{2}$ & ${ }^{1} A$ \\
\hline
\end{tabular}

however, that for a planar configuration, the bond order is too high.

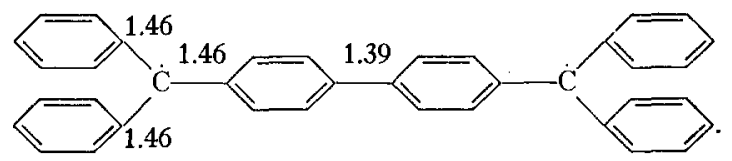

Even for the biphenyl configuration (maximum nonplanarity of the diarylmethyl groups), the bond order of the phenylene-phenylene bond, 1.21 , is too large for free rotation. It is interesting to note that the order of the methyl carbon bonds is unchanged from that of triphenylmethyl.

Another mechanism must be found, therefore, which will decouple the spin from the orbital momentum of the electron. MO calculation on compound IIc shows that the lowest triplet must possess the symmetry representation ${ }^{3} B_{1 u}$ for a planar molecule $\left(D_{2 h}\right.$ symmetry) and ${ }^{3} B_{1}$ if the molecule is reduced to $D_{2}$ symmetry by the steric interference of the phenyl rings. Table I shows the singlet states which can be combined with the lowest triplet state through the various terms of the spin-orbit interaction.

Except for the ground state in the molecule with $D_{2}$ symmetry, the singlet states of the proper symmetry for interaction are probably so far removed that their spin orbit contribution to the lowest triplet is easily decoupled by the tumbling of the molecule. The only spin orbit contribution which could be appreciable is that arising from the ground state singlet. However, Mizushima and Koide ${ }^{12}$ have shown that the matrix elements involving $S_{z}$ are zero. Contributions to the matrix element in $S_{2}$ may arise when some of their approximations are taken into account, but these terms are small and should also average out with molecular tumbling. For triplet states which lie high in energy, the spin-orbit contribution from $S_{ \pm}$may be appreciable; and molecular tumbling will not average out the interaction.

Compound IIa does not exhibit resonance absorption either in the solid state or in solution. MO calculation indicates that the energy separation between the ground state and the triplet state is $6.1 \mathrm{kcal}$. Therefore, $7.5 \times 10^{-30} \%$ of the molecules should be in the triplet state at room temperature. This low concentration of radicals plus the low solubility of the compounds leads to a quantity of radicals in solution which is below the detectability of the paramagnetic resonance equipment.

A barely detectable resonance was observed in the tetraxenyl analog (IIb) at $g=2.0025 \pm 0.0004$ after the

${ }^{12}$ M. Mizushima and S. Koide, J. Chem. Phys. 20, 765 (1952). compound was dehalogenated for a three-day period in the reaction vessel. MO calculation indicates that the triplet state of this compound should lie approximately $5.7 \mathrm{kcal}$ above the ground state. Hence, the additional chemical resonance of the xenyl groups does not lower significantly the position of the triplet state below that of compound IIa. Possibly the very low solubility of the compound in benzene resulted in slow dehalogenation by the silver and the resonance observed was caused by a monoradical formed after the removal of only one chlorine.

The intensities of the absorptions in the compounds in group II were also observed to diminish at rates comparable to those in group I. The degraded compounds appear to retain their original colors when stored in evacuated tubes even after the resonance absorption has disappeared. It has been found ${ }^{13}$ that the optical spectra of certain free radicals change during degradation without change in the observed color. On exposure to air, the residual color is totally dissipated.

\section{Magnetic Resonance Spectrum of Group III Compound}

The unpaired electrons in the ether derivative are not as well "insulated" from one another as in the compounds of group I because of the existence of a $\pi$ orbital on the oxygen. Therefore, the molecule should possess a diamagnetic ground state as in the compounds of group II. Figure 4 shows the resonance absorption spectrum observed for this biradical. The hyperfine structure is as well resolved as in the methylene derivative of group I. The individual peaks are sharper and more closely spaced so that the line width for the ether is 7.6 gauss as compared to 11.3 gauss for the methylene derivative. The $g$-value for this compound is 2.0031 \pm 0.0004 . This value, which is greater than the $g$-values in the other compounds, indicates that the spherically symmetrical component of the spin-orbit coupling is larger. An MO calculation on the planar compound

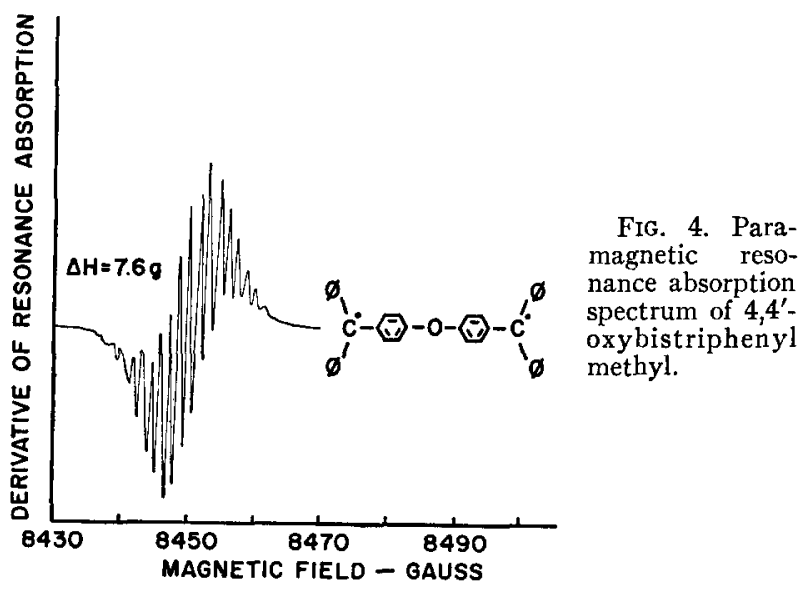

${ }^{13}$ P. W. Selwood and R. F. Preckel, J. Am. Chem. Soc. 65, 895 (1943). 
shows that the first excited state lies only $480 \mathrm{cal}$ above the ground state. Therefore, about $75 \%$ of the molecules should exist in the triplet state at room temperature. It is not unreasonable then that the $g$-factor is larger since the ground state of this molecule can combine with the triplet state through spin-orbit interaction.

Fresh solutions of the ether show strong resonance absorption; but, as in other compounds, this absorption gradually disappears, with the accompanying appearance of a light yellow precipitate, probably the cyclic dimer described by Müller. ${ }^{14}$

\section{DISCUSSION}

The appearance of paramagnetic resonance absorption in the compounds discussed above is clear evidence that a paramagnetic species occurs in benzene solutions of these materials. Whether the compounds possess two unpaired electrons could be decided only by a quantitative determination of the paramagnetic susceptibility of the radicals from the area under their resonance absorption curves. Where resonance is detectable, the population of the molecules in the triplet state is at least one percent. Intensities of absorption in groups I and III compounds are larger than in group II, indicating a considerably larger population of the triplet in these compounds.

The observation of hyperfine structure in the resonance spectra of free radicals in solution deserves further consideration. The presence of this hyperfine structure leads to the conclusion that there are terms in the hyperfine interaction which are not averaged out by the rapid tumbling of the molecules. ${ }^{9}$ Such a spherically symmetrical contribution would give rise to a spin Hamiltonian of the form, $\bar{S} \cdot \sum_{i} a_{i} \bar{I}_{i}$, where $\bar{S}$ and $\bar{I}$ are the electron and nuclear spin operators, respectively. The summation is over the nuclei contributing to the hyperfine structure and $a_{i}$ gives the magnitude of the hyperfine interaction between the unpaired electron and $i$ th hydrogen nucleus. Since the hyperfine Hamiltonian depends strongly on the distance from the hydrogen nucleus, $a_{i}$ to a good approximation is dependent on the unpaired electron density in the immediate vicinity of the carbon atom nearest the $i$ th hydrogen. As discussed above, if the biradicals of group I may be con-

\footnotetext{
${ }^{14}$ E. Müller and W. Bunge, Ber. deut. Chem. ges. 69, 2164 (1936).
}

sidered as two monoradicals with independent unpaired electrons, the summation may be carried out over only one half of the biradical. This assumption does not greatly simplify fitting the spin-Hamiltonian to the experimental data, however. For example, if the orthopositions of each ring possess the same electron density and if similar assumptions are made for the meta- and para-positions, the spin-Hamiltonian becomes

$$
\bar{S} \cdot\left[a_{\text {ortho }} \sum_{\text {ortho }} \bar{I}_{i}+a_{\text {meta }} \sum_{\text {meta }} \bar{I}_{i}+a_{\text {para }} \sum_{\text {para }} \bar{I}_{i}\right] .
$$

Since the three rings probably do not lie in a plane, ${ }^{8}$ there is no reason to assume the $a$ 's are equivalent and 147 possible hyperfine structure lines result. In addition, because of the nonplanarity of the rings, the electron distribution cannot be determined reliably by simple molecular orbital theory. If planarity is assumed, $a_{\text {meta }}=0, a_{\text {ortho }}=a_{\text {para }}$, and only 9 hyperfine lines would be observed. Obviously, from the experimental results, any assumption of planarity is not valid.

Some clarification is necessary since the assumption of planarity (which is used because it greatly simplifies the MO calculations) has been made throughout this paper. The fact that the molecules are not planar does not invalidate the method of the calculations, but alters the results to some extent. For example, in triphenylmethyl, if nonplanarity is assumed, (a propeller-shaped molecule with $D_{3}$ symmetry), the exchange integral between the methyl carbon and the phenyl rings is decreased. This effect results in a decrease in the resonance energy, as calculated by MO theory, below the value calculated for a planar molecule. ${ }^{15}$ Reduction of this exchange integral to $0.83 \beta$ brings the MO calculation into agreement with experiment. Unfortunately, this configuration of the radical does not account for all the discrepancies between experiment and theory since the ratio of the $a_{i}$ 's in the foregoing spin-Hamiltonian remain unchanged. This, of course, may be a fault of the simplifying approximation used in the MO approach. In any event, coplanarity is not necessary for chemical resonance stabilization but is required only for a maximum resonance energy. Therefore, the biradicals discussed above are highly resonance stabilized even though coplanarity is not possible because of the steric factors.

${ }^{15}$ E. Huckel, Z. Physik 83, 632 (1933). 